\title{
An Approach for Self-Adaptive Path Loss Modelling for Positioning in Underground Environments
}

\author{
Evgeny Osipov, ${ }^{1}$ Denis Kleyko, ${ }^{1}$ and Alexey Shapin ${ }^{2}$ \\ ${ }^{1}$ Department of Computer Science, Electrical and Space Engineering, Luleå University of Technology, 97187 Luleå, Sweden \\ ${ }^{2}$ Department of Automatic Electrical Communication, Siberian State University of Telecommunications and Information Sciences, \\ Novosibirsk 630102, Russia \\ Correspondence should be addressed to Denis Kleyko; denis.kleyko@gmail.com
}

Received 3 November 2015; Revised 27 January 2016; Accepted 28 January 2016

Academic Editor: Jose-Maria Molina-Garcia-Pardo

Copyright (C) 2016 Evgeny Osipov et al. This is an open access article distributed under the Creative Commons Attribution License, which permits unrestricted use, distribution, and reproduction in any medium, provided the original work is properly cited.

\begin{abstract}
This paper proposes a real-time self-adaptive approach for accurate path loss estimation in underground mines or tunnels based on signal strength measurements from heterogeneous radio communication technologies. The proposed model features simplicity of implementation. The methodology is validated in simulations and verified by measurements taken in real environments. The proposed method leverages accuracy of positioning matching the existing approaches while requiring smaller engineering efforts.
\end{abstract}

\section{Introduction}

Accurate positioning in underground environments like tunnels or mines is essential for their efficient operation (e.g., logistics, energy savings, and improved decision support) as well as for personal safety, for example, reducing the number of incidents. For example, in the mining industry there is a need for positioning of people, equipment, and vehicles. At the same time precise positioning based on the properties of the radio signal propagation requires accurate characterization of the radio channel, which is challenging to generalize for a given technology, due to the unique geometry of the corridors as well as the reflective properties of the walls' material. This paper elaborates on the feasibility of designing a positioning service built upon the existing standard wireless infrastructure deployed in mines. Wireless communication networks became an integral part of the mining process nowadays: Several large mines, for example, in Canada [1], Sweden [2], and Bulgaria [3], have deployed underground IEEE 802.11 based networks covering all tunnels. However, Wi-Fi systems alone cannot be considered for accurate positioning. Therefore the main motivation for this work is that other radio systems with higher accuracy in combination with IEEE 802.11 networks should jointly enhance the accuracy of positioning. An example of such a complementary system is RFID with passive tags, which is often installed for safety monitoring, monitoring of assets and environmental conditions, controlling of delivery cycles of trucks, collision avoidance between vehicles in tunnels, and so forth. Several companies provide and deploy RFID solutions worldwide (see [4] for more details on the RFID deployment in Australia, Chile, India, and Sweden). This paper presents a methodology, which utilizes the diversity of the deployed radio technologies and provides a robust and accurate positioning service by combining information from multiple heterogeneous wireless technologies.

The traditional methods of communication system-aided positioning include time-of-arrival, angle-of-arrival, and received signal strength indication (RSSI) approaches [5]. The key aspect in all these approaches is the transformation of the signal strength level into the actual distances. The decisive factor influencing the accuracy at this step is the quality of the adopted radio propagation model and the ability to accurately estimate the radio signal path loss in the given environment. While in most of outdoor scenarios the simple free space path loss (FSPL) model [6] provides sufficient accuracy, positioning process in underground scenarios is challenging due to complex electromagnetic characteristics of the environment. In underground environment the signal propagation characteristics depend on many factors, for example, the surface of 


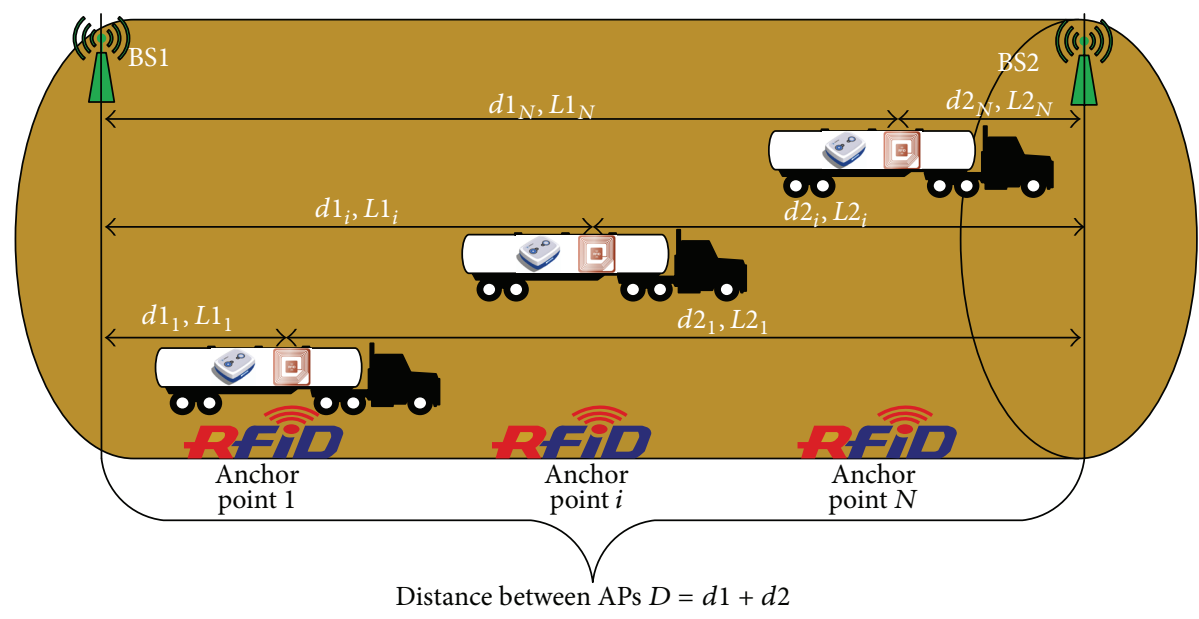

FIGURE 1: System model.

a tunnel, the geometry of the tunnel, the type of a positioning object (a car, a truck, or a human), the presence of obstacles between the receiver and the transmitter, and the presence of mobile objects.

Practically it is known that that there is no good enough analytical model for describing all possible variations of the physical topologies. This paper proposes a practical approach for self-adaptive estimation of the signal path loss in the given deployment site. The main contribution is formulated as a method for self-adaptive template path loss model based on aggregation of data from heterogeneous wireless technologies.

The paper is organized as follows. An overview of the related work is provided in Section 2. Section 3 describes the system-level architecture. Section 4 presents self-adaptive iterative path loss model. Section 5 provides validation of the proposed methodology and its real-life performance. Finally, Section 6 discusses and concludes the paper.

\section{Related Work}

The current state of the art of wireless communications and propagation modelling in underground mines can be found in $[7,8]$. The existing methods can be classified as numerical methods for solving Maxwell equations [9, 10]; waveguide based channel models [11, 12]; Ray tracing based channel models [13]; and empirical path loss modelling. The approach presented in this paper falls in the latter domain. The main models are so-called $N$-slopes models, where the signal attenuation is modelled differently on $N$ discrete intervals from the source of the radio signal. For instance, [14] proposes a four-slope path loss model for car tunnels. The work in [15] further extends the model into a five-zone model for the scenario when the size of object is comparable with the size of the tunnel and the transmission range could be up to several thousand meters. For this paper we adopt the two-piece path loss model [16], which features computational simplicity while being known for adequate accuracy. We argue that even the simplest model can leverage competitive performance when supported by information from several communication technologies.

\section{System-Level Architecture Description}

Consider a generic communication system to be deployed in an underground environment as illustrated in Figure 1. The system deploys heterogeneous wireless technologies: IEEE 802.11 based infrastructure network and a near-field communication (NFC) system. The IEEE 802.11 based network consists of base stations (BSs) and transceivers installed on different mobile and static objects, for example, vehicles, workers, and facilities. The near-field communication system consists of RFID tags and RFID readers. In mines the NFC is used for various purposes including access control and inventory of objects. The coordinates of both IEEE 802.11 based BSs and RFID readers are known exactly.

Note that in reality several wireless networks (also from different vendors) could be deployed on orthogonal channels for serving different purposes, for example, a network for VoIP and personal data communications and a network for remote control of automatic vehicles. This paper, however, considers a generic case of a single production network with virtualization capabilities connected to the main control room. Thus, it is assumed that all base stations are interconnected by a wire line network forming an extended service set. It is also assumed that the RFID readers are connected to the same production network either wirelessly or directly. Therefore, all information from all connected devices is available centrally.

The client side of the system is represented by RFID tags and different kind of IEEE 802.11 based devices including data communication units (personal communicators, devices for remote control of vehicles, etc.) as well as Wi-Fi based asset tags [17]. The latter devices are specifically designed for WiFi based positioning. The number of deployed RFID readers between two base stations is $N_{\text {RFID }} \in[0, \ldots, N]$ (anchor points): while some tunnels could be equipped with several RFID readers, other tunnels might lack any at all. 


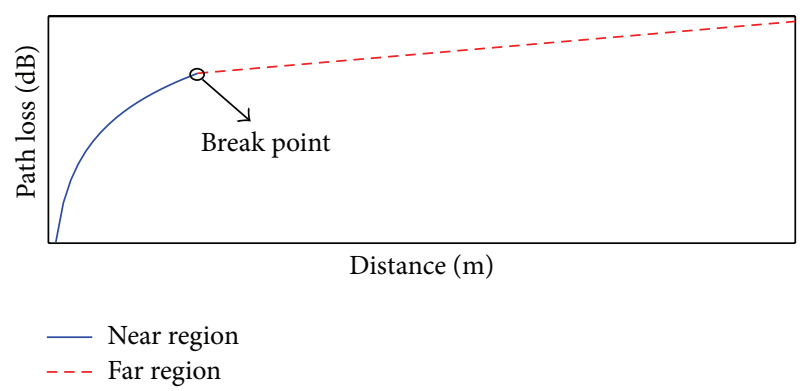

FIgURE 2: Two-piece model.

3.1. Outline of the Solution. The proposed solution addresses one of the fundamental challenges of accurate modelling of radio signal path loss in the underground environment. The main idea is to adopt the simplest possible model of signal path loss with distance (two-piece model in Figure 2) with the initially unknown parameters. Despite its simplicity the model captures the major radio propagation modes. In the near region, the path loss slope is steep and in many cases it is modelled as free space path loss. In the far region, the waveguide effect appears with few lower order modes and the path loss slope is reduced significantly [8]. This model will be used as a template for the target model to be used for RSSI-based positioning at Wi-Fi base stations. Note that while fading is an important effect for wireless propagation models it is not accounted by the adopted two-piece template. The aim of the proposed methodology is to study the feasibility of accurate positioning with a simple model in a real-life underground environment. Overall the goal is formulated as to come up with accurate and tunnel-specific estimates of the path loss by overlaying the events of measuring the signal strength from a particular node at the base station with the events when this monitoring node is engaged with one of the RFID readers. This will be done by constructing and solving a system of 2 . $N_{\text {RFID }}$ equations describing the model and the corresponding signal strength measurements $L_{i}$ in Figure 1 at points of engagement with RFID readers $\left(d_{i}\right.$ in Figure 1$)$. The system of equations will be solved iteratively, where the iteration is a passage of an object through all RFID readers in the particular tunnel.

The resulting model could be used then in two ways. Firstly, in tunnels featuring RFID readers the model will be continuously updated accounting for dynamically changing properties of the propagation environment due to mobility of the monitored nodes. In this case the accuracy of the estimated model will be the highest one. Secondly, the averaged model will be used statically in corridors lacking RFID technology, but having similar geometry. In this case the accuracy of the model will be lower than in the previous case; however, it will be higher compared to the case when model's parameters are chosen according to commonly used assumptions without anchoring to the specifics of the particular operation environment.

\section{Self-Adaptive Path Loss Model}

This section presents the details of the proposed methodology. First, the iterative model construction is presented. Then the operation phase is discussed.

4.1. Iterative Model. As argued earlier for simplicity of the engineering the two-piece model [8] is adopted. The twopiece model defines two (near and far) regions. The path loss in the near region is modelled by the free space path loss model (1), where $\gamma$ is the path loss exponent, $C$ is the constant, which takes into account system losses, and $d$ is the distance between the transmitter and the receiver. $L$ is the path loss in $\mathrm{dB}$. The waveguide effect, which reduces the path loss, is captured by the model of the far region. The path loss in the far region is modelled by a linear approximation (2) [14], where $\alpha$ is the curve slope and $L_{0}$ and $d_{0}$ are the path loss and distance at the break point, respectively:

$$
\begin{aligned}
& L[\mathrm{~dB}]=\gamma \cdot\left(10 \log _{10}(d)+C\right), \\
& L[\mathrm{~dB}]=L_{0}[\mathrm{~dB}]+\alpha \cdot\left(d-d_{0}\right) .
\end{aligned}
$$

The break point $d_{0}$ divides the whole distance into two regions. Note that the estimation of the break point position is one of the key issues in the process of two-piece model construction. There are several empirical and analytical methods solving this problem (please see the discussion in [8] for more details). For example, the four-slope channel model in [14] estimates the break point $d_{0}$ (the first break point in the fourslope model) using a first Fresnel zone calculated as in [14], which requires heights of receiver and transmitter antennas. Because the proposed approach assumes no a priori knowledge about the heights of antennas above the ground this method cannot be used. Instead we adopted the method proposed in [18] which defines the break point as the point where propagation losses from both models are equal. Thus, the break point position is the adjustable parameter for the proposed approach.

Summarizing the above discussion the two-piece model, which we further on refer to as the template model, is presented in

$$
\begin{aligned}
L[\mathrm{~dB}] & =\gamma \cdot\left(10 \log _{10}(d)+C\right), \quad \text { when } d \leq d_{0}, \\
L[\mathrm{~dB}] & =L_{0}[\mathrm{~dB}]+\alpha \cdot\left(d-d_{0}\right), \quad \text { otherwise, } \\
L_{0}[\mathrm{~dB}] & =\gamma \cdot\left(10 \log _{10}\left(d_{0}\right)+C\right) .
\end{aligned}
$$

The template model has four unknown parameters, namely, $\gamma, C, d_{0}$, and $\alpha$. These parameters will be estimated and tuned iteratively upon collecting the data from heterogeneous wireless sources. Now, each time the RFID and the Wi-Fi enabled node passes by RFID reader (an anchor point) it also communicates with neighbouring base stations.

According to the system model in Figure 3 the object is located between two BSs and distance $D$ between BSs is known. When the object passes by the $i$ th RFID reader its distances to base station $1\left(d 1_{i}\right)$ and base station $2\left(d 2_{i}\right)$ are determined. At the same time BSs measure RSSI of the ping pulses issued by the object; that is, path losses $L 1_{i}, L 2_{i}$ are 


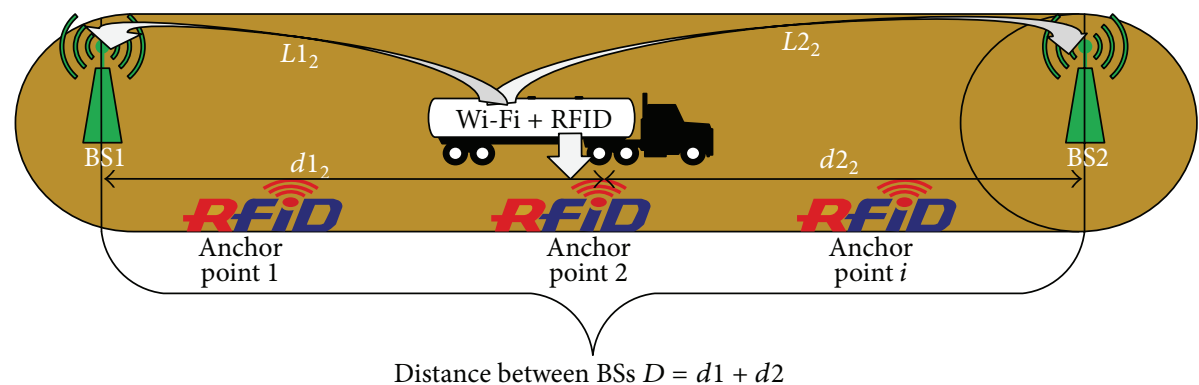

FIgURE 3: An example of the system model when the object (illustrated by the truck) is passing by RFID reader 2.

known as well. Thus, the system of (4) is constructed for each RFID reader, where $i$ is the $i$ th reader:

$$
\begin{array}{ll}
L 1_{i}[\mathrm{~dB}]=\gamma \cdot\left(10 \log _{10}\left(d 1_{i}\right)+C\right), & \text { when } d 1_{i} \leq d_{0}, \\
L 1_{i}[\mathrm{~dB}]=L_{0}[\mathrm{~dB}]+\alpha \cdot\left(d 1_{i}-d_{0}\right), & \text { otherwise, } \\
L 2_{i}[\mathrm{~dB}]=\gamma \cdot\left(10 \log _{10}\left(d 2_{i}\right)+C\right), & \text { when } d 2_{i} \leq d_{0}, \\
L 2_{i}[\mathrm{~dB}]=L_{0}[\mathrm{~dB}]+\alpha \cdot\left(d 2_{i}-d_{0}\right), & \text { otherwise. }
\end{array}
$$

During the runtime system of up to $N_{\text {RFID }}$ systems of equations of form (4) is constructed at each iteration. The system is then solved numerically, for example, using nonlinear least-squares method [19].

4.2. Operating Phase. When the four parameters for the template model are estimated the distance from an object to the BS can be calculated as in

$$
\begin{aligned}
& d=10^{(L[\mathrm{~dB}] / \gamma-C) / 10}, \quad \text { when } L[\mathrm{~dB}] \leq L_{0}[\mathrm{~dB}], \\
& d=d_{0}+\frac{L[\mathrm{~dB}]-L_{0}[\mathrm{~dB}]}{\alpha}, \quad \text { otherwise. }
\end{aligned}
$$

Note that when the object is located between two base stations as in Figure 1 the additional condition such as the distance between base stations $D$ should be considered. Obviously, due to errors in the estimation of $d 1$ and $d 2$ their sum may be lesser or larger than distance $D$. Therefore, the obtained values are normalized on $D$ with respect to (6). One should consider that normalization is appropriate only if the object is positioned between base stations. Moreover, the estimated model will work even in the case when there is a signal level only from one BS; that is, the normalization is impossible:

$$
\begin{aligned}
& \widetilde{d 1}=D \cdot \frac{d 1}{d 1+d 2}, \quad \text { when } d 1+d 2>D \text { or } \\
& \widetilde{d 2}=D \cdot \frac{d 2}{d 1+d 2}, \quad \text { when } d 1+d 2<D .
\end{aligned}
$$

Thus for the system in Figure 3 in order to form the model and subsequently estimate the distances the following actions are needed:

(i) Choose template model (3).

(ii) Detect that the object has passed $i$ th RFID reader. (iii) Calculate distances $d 1_{i}$ and $d 2_{i}$ from the reader to the base stations.

(iv) Measure path losses $L 1_{i}$ and $L 2_{i}$ of the object's signal at the base stations.

(v) Construct a system of equations in the form (4).

(vi) Repeat the above steps for $N_{\text {RFID }}$ readers in the tunnel.

(vii) Estimate the parameters for the template model by solving the system of equations, for example, using nonlinear least-squares method [19].

(viii) Calculate distances $d 1$ and $d 2$ from the object to the bases stations according to (5).

(ix) Where it is applicable normalize the distances as in (6)

\section{Validation of the Proposed Methodology and Its Real-Life Performance}

For the validation of the proposed methodology of the signal path loss and its convergence properties the following approach was adopted. The two-piece model with arbitrary chosen parameters is calculated for stretch [0-300] meters and used as the reference case. The reference model's parameters were set as $\gamma=2$ and $C=20.1$ and $d_{0}=50$ meters and $\alpha=0.2$. Note that this choice of parameters' values does not correspond to any particular operating environment. While the model for the validation might at the first glance seem unjustifiably similar to the template model it is a valid choice for the pragmatic validation approach, that is, to check first whether the proposed approach is viable for at least an artificial model with similar structure and known parameters. Next the assessment of the approach in real-life environments is presented in the subsequent subsections.

The training set for the proposed iterative method is constructed by first sampling the calculated values of the twopiece reference model in $N$ anchor points ( $N$ will be varied in the simulations). In order to reflect the fact that real RSSI values are noisy the additive white Gaussian noise was randomly added to each sampled value resulting in SNR value being $-2 \mathrm{~dB}$ on average and the standard deviation of the noise was $1.25 \mathrm{~dB}$. This is reflected by the red curve in Figure 4. Note that SNR value was chosen to be low in order to demonstrate that the parameters for the template model can be estimated even in noisy environment. 


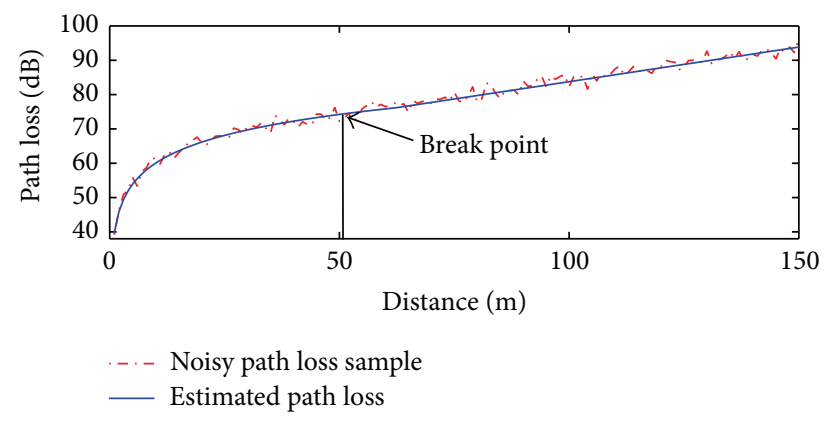

FIGURE 4: Path loss of noisy signal sample and estimations made by the proposed method using four non-uniformly distributed anchor points.

The task of validation is formulated as to apply the proposed method on the noisy sequence of RSSI values and then to estimate the parameters for the template model and finally compare the estimated values to the parameters of the reference model.

The model parameters were estimated in four simulations for different number of anchor points $N \in[19,14,9,4]$. In the simulations with $N=19, N=14$, and $N=9$ the distances between points were distributed uniformly on a stretch of $300 \mathrm{~m}$. The simulation with $N=4$ was conducted with the nonuniform placement of the anchor points by reason described below. In this simulation the anchor points were set on $15,30,270$, and 285 meters.

Each simulation for a given number of anchor points consisted of 100 iterations. At each iteration one RSSI sample with $N$ values was generated. At each iteration the samples from the previous iterations were added to the training set and the average value of path loss $\bar{L}$ was calculated for each anchor point. This is done in order to understand the accuracy and the convergence time properties of the proposed self-adaptive path loss estimation methodology.

Figure 4 shows an example of results obtained from the iteration of a simulation with 100 samples for four anchor points. The red dashed curve shows one of noisy samples used in this iteration and the solid curve shows the two-piece model computed with the estimated model by the proposed method parameters.

5.1. Convergence and the Factors Affecting the Accuracy. Figure 5 illustrates the development of the four estimated parameters with the increasing number of samples taken into consideration and different number of anchor points considered in the simulation. The main observation concerns the convergence property of the proposed estimation method. It is easy to see that all four estimated parameters converge differently to the values of the reference model for different number of the anchor points. While with 19 anchors the parameters estimated by the proposed self-adaptive method (the red curves in the figure) converge to the target values (the straight line in the figure) fairly quickly, the convergence time worsens when the number of anchors is decreasing. With 9 anchors the proposed method does not converge to the reference values. This is because with uniform distribution of the small numbers of anchors over the monitored stretch most of them fall into the far region of the two-piece model. With 19 anchors distributed evenly over the same stretch the numbers of nodes in both the near and the far regions are sufficient for the estimation. The large number of anchors is, however, not practical to have from the economical point of view.

We, therefore, simulated a case with the nonuniform placement of the anchor points. Specifically, the points in the four anchors' simulation were set on 15, 30, 270, and 285 meters. With this placement two nodes were placed in the presumed near region of the base station at the beginning of the stretch and other two anchors were placed in the presumed near region of the base station at the far end of the stretch. For any of the two base stations the other two nodes were located in their corresponding far region. The results of the simulations (shown by the magenta coloured curves in Figure 5) show a very good convergence of all estimated parameters to the reference values.

This observation gives an important insight into the rules for the placement of the RFID readers in the underground environment: If the RFID readers are intended to be used for calibration of the Wi-Fi based positioning system, their limited number should be placed in a relative proximity to the base station, that is, in the presumed near region of the transmitter. Observed results promise that the approach will also be viable in a real-life underground environment.

\subsection{Assessment of the Estimation Accuracy in Real Environ-} ments. The accuracy of the proposed self-adaptive path loss estimation model was assessed in two real-life operating environments. The first environment is 80 -meter underground corridor in the university building. The second environment is two 300-meter-long tunnels in ore mine in northern Sweden. The following subsections report the results of these experiments.

5.3. Method's Accuracy in the Underground Corridor. The length of the considered corridor is approximately 80 meters. The corridor has a rectangular shape with the following dimensions: width of 1.8 meters and height of 2.6 meters. The measurements were taken using three laptops. Two of them were located at distances 0 and 80 meters and played roles of base stations. The third laptop was used for imitation of the moving object. The RSSI values from the base station were taken with 2 -meter interval. Thus measurements from 38 points were used to obtain the estimate of the path loss model parameters. Note that the 38 locations here are used for testing purposes only and this number does not indicate the number of needed RFID readers. On the contrary the results of the validation show that only few readers are sufficient for the estimation of parameters. The measurements and the result of the estimation are depicted in Figure 6. The red circles in the figure denoted as RSSI $\mathrm{BS1}_{1}$ in the legend correspond to the measurements taken at the base station on the one end of the corridor and blue crosses, denoted as $\mathrm{RSSI}_{\mathrm{BS} 2}$, reflect the RSSI values measured by the base station at the other end of the corridor. 

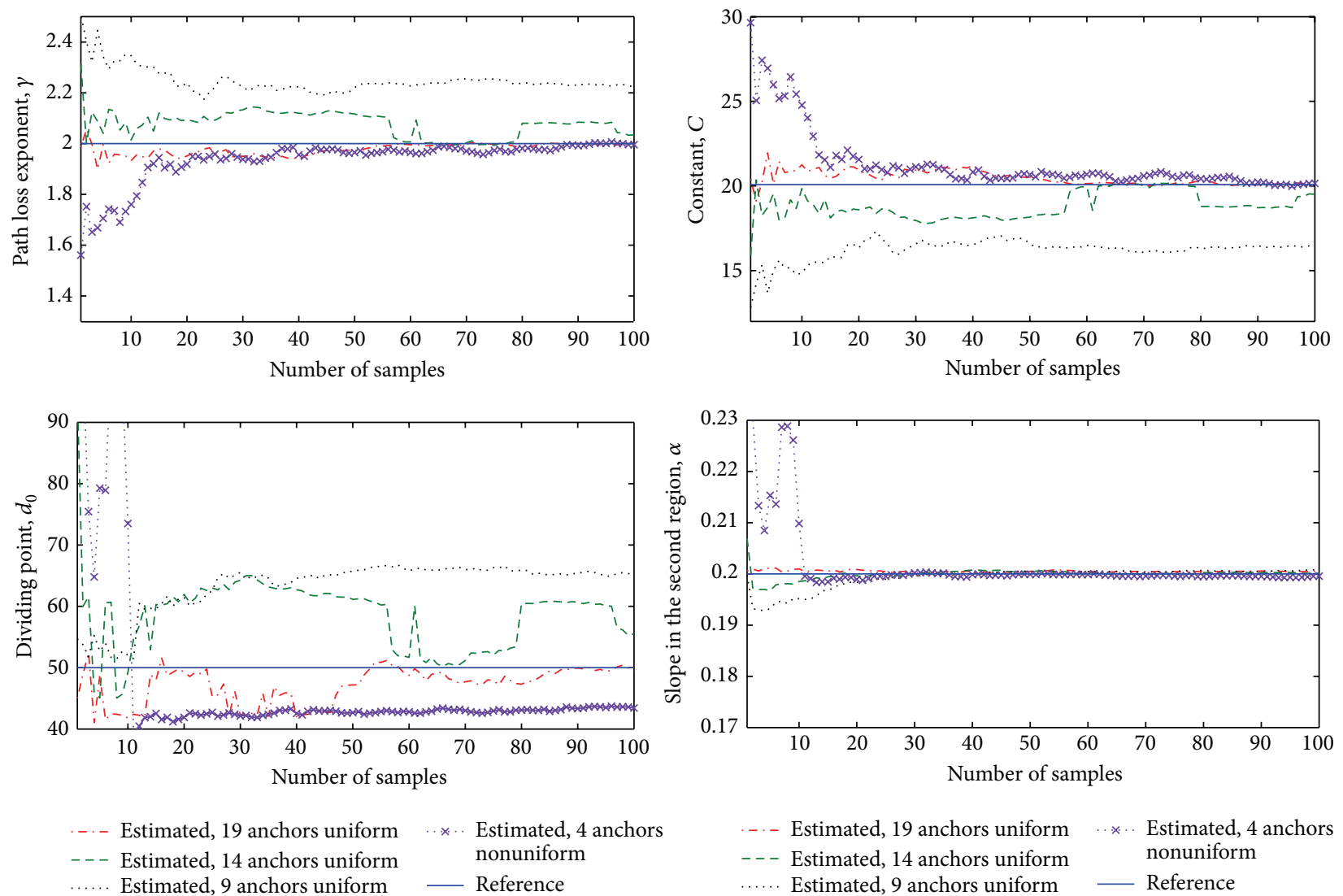

Figure 5: Dynamics of the estimated parameters.

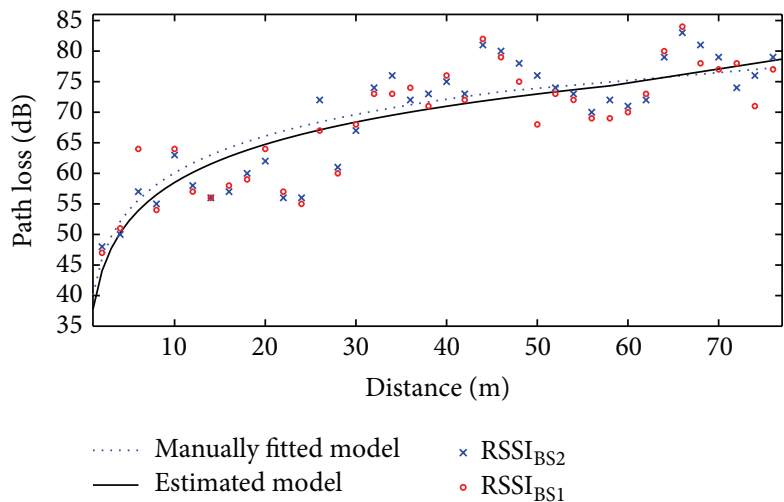

FIGURE 6: Comparison between hand calculated and estimated models.

In order to assess the performance of the proposed method a reference model was calculated using the parameters of FSPL model in the near region, the break point was calculated as the first break point in [14], and the slope for the far region was fitted based on the measured values. This reference model is illustrated by the blue dotted curve in Figure 6. The model based on the estimates of the parameters from the proposed iterative model is shown by the solid black curve in the figure. The graph shows no significant difference between the model based on the method estimated by the

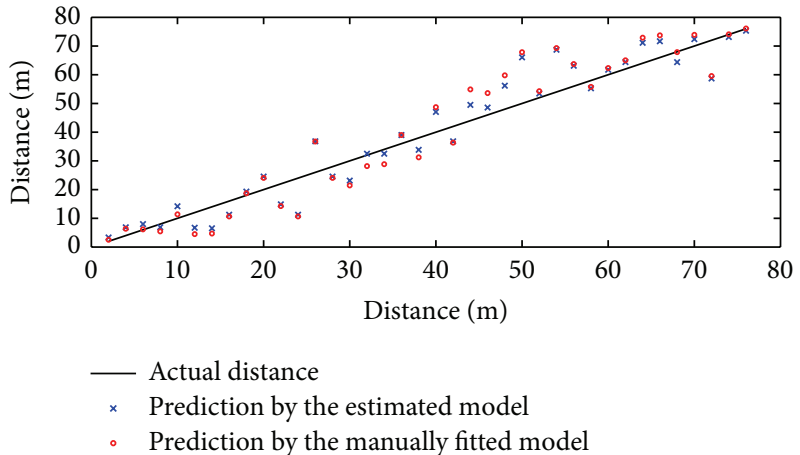

Figure 7: Predicted distances by hand calculated and estimated models.

proposed methodology model and the best one manually fitted to the measurements.

Note that in the operating phase the proposed model is used for the estimation of the distance to the BS. Hence it is important that the distance predictions by the proposed model are similar to predictions from the hand calculated model. The actual distances and the results of prediction by the models normalized with (6) are depicted in Figure 7. The actual distances are shown by the solid black curve in the figure. The blue crosses in Figure 7 correspond to the distance predictions made by the model with parameters estimated by 


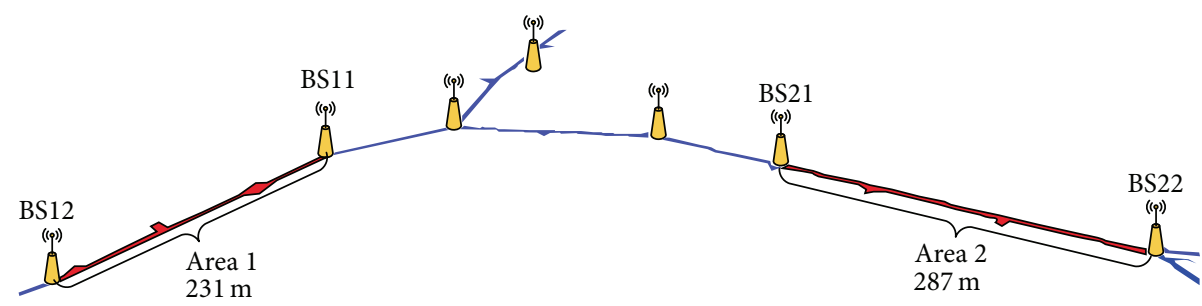

FIGURE 8: The areas for the experiments (only areas in red are considered).

the proposed approach. The red circles reflect the distance predictions made by the reference manually fitted model. These results show that the estimated model and the reference model have the same level of accuracy to the measurements. However, there is no need to do manual calculations and know the deployment in the environment for the estimation of model parameters with the proposed approach.

5.4. Method's Accuracy Assessed in the Ore Mine. This time the model was assessed in Kristineberg ore mine [20] located in northern Sweden. The mine is equipped with IEEE 802.11based network from Cisco operating in $2.4 \mathrm{GHz}$ band. On the testing site most of the mine's corridors are covered by WiFi network. Wi-Fi access points are deployed such that the objects are visible by at least two access points (see a segment of the mine in Figure 8). Thus, communications are mainly line-of-sight except for some tunnels, which are curved. The shape of corridors is close to circular with the approximate diameter of 6 meters. The training set was obtained by recording RSSI level in ping pulses issued by Ekahau asset tags [17]. Figure 8 shows the layout of mine's areas, where the measurements were taken.

During the measurements the tunnels were empty. Since the mine currently is not equipped with RFID readers, measurements were taken manually similarly to the corridor scenario. The distances between anchor points were uniformly distributed with 15-meter intervals. RSSI values on base stations were stored and associated with the anchor points. All anchor points were used for the template path loss model estimation.

The proposed approach was applied to the collected measurements. The measurements campaign was done in Area 1 and Area 2. The assessment procedure is similar to the corridor environment. Figures 9 and 10 demonstrate the measurements and the results of the estimations made by the proposed approach for Area 1 and Area 2, respectively. The accuracy of the distances is similar to the one shown in the corridor environment. Therefore, the graphical representation of the results is omitted for space saving reasons.

The blue crosses in the figures denoted as $\mathrm{RSSI}_{\mathrm{BS} 11}$ or $\mathrm{RSSI}_{\mathrm{BS} 21}$ in the legends correspond to the measurements taken at the base stations at the beginning of the stretches. Red circles, denoted as $\mathrm{RSSI}_{\mathrm{BS} 12}$ or RSSI ${ }_{\mathrm{BS} 22}$, reflect the RSSI values measured by the base stations at the far ends of the areas. The figures show that in both cases the models with the estimated parameters fit the provided measurements.

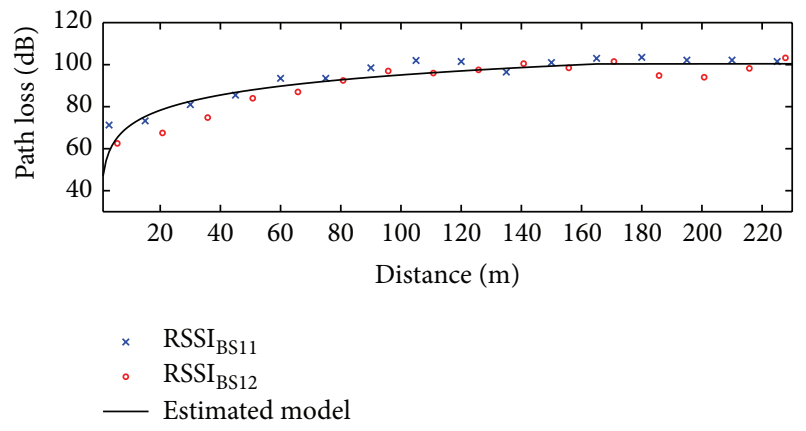

Figure 9: Two-piece estimated model for measurements in Area 1.

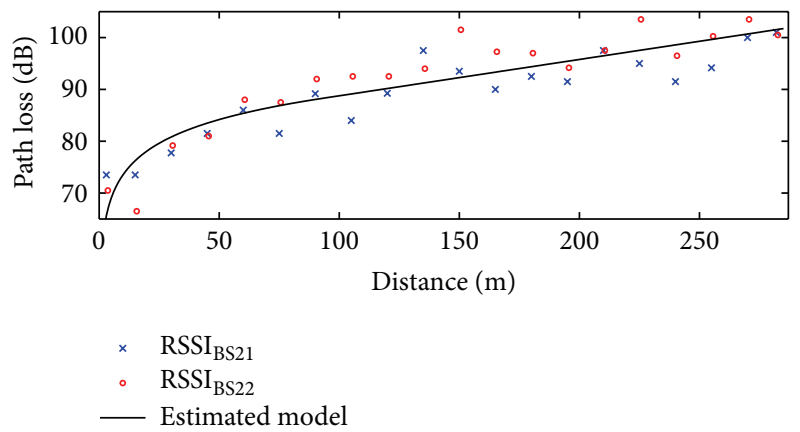

FIGURE 10: Two-piece estimated model for measurements in Area 2.

\section{Discussion and Conclusion}

This paper proposed a simple practical approach for the selfadaptive estimation of the parameters for the two-piece path loss model. The estimated parameters accurately reflect the signal attenuation in the particular indoor or underground monitored environment. It is proposed to build an accurate Wi-Fi based positioning system using the simple two-piece path loss model with the estimated parameters. The proposed approach is self-adaptive in a sense that it does not require manual engineering of the model parameters. Instead, using the data from heterogeneous wireless technologies the model's parameters converge to the environment-specific values in several iterations. The accuracy of the proposed approach obviously increases as the history of RSSI measurements grow, that is, with the number of estimation iterations.

Along the side of the validation process a procedure for placing the anchor points was developed. Namely, the 
quantity of the anchor points itself plays secondary role compared to the actual placement of RFID anchors. It is important to insert anchor points in both the presumed near and far regions of the two-piece model. In this case even very few anchor points per the monitored region are sufficient for accurate estimation of the path loss parameters.

On the level of a discussion the larger number of anchor points could be used to create a piecewise linear model of path loss on each interval between the RFID anchors. The development of this idea and its accuracy evaluation are subjects for future work. Another future work item concerns recognizing patterns of objects' mobility in the underground tunnels. Also particular resources should be dedicated for studies on how the effects such as shadow fading could affect the resulting models. Besides, the presence of vehicles obviously introduces additional impairments to radio signal propagation. On the other hand the information about the type of the moving object which is logged in the central control and supervision system could be overlaid with the RSSI measurements for further improvement of the positioning accuracy.

\section{Conflict of Interests}

The authors declare that there is no conflict of interests regarding the publication of this paper.

\section{Acknowledgments}

This work is supported by the Swedish Governmental Agency for Innovation Systems (VINNOVA), under Project 201300265 Industrial Positioning System. The authors also thank Mobilaris company for the assistance while performing measurements in the mine.

\section{References}

[1] H. Solomon, "How a Canadian mining company put a Wi-Fi network underground," 2013, http://www.itworldcanada.com/ article/how-a-canadian-mining-company-put-a-wi-fi-networkunderground/87122.

[2] Mine of information. ABB review 2013, https://www.controlglobal.com/assets/knowledge_centers/abb/assets/ABB-Review2-2013-Mine-of-information.pdf.

[3] Underground Mine Communications and Monitoring, http:// im-mining.com/2012/05/01/underground-mine-communications-monitoring/.

[4] RFID Journal, "How is RFID Being Applied in the Mining Sector?” http://www.rfidjournal.com/blogs/experts/entry?10649.

[5] A. Kupper, "Fundamentals of positioning," in Location-Based Services Fundamentals and Operation, chapter 6, pp. 123-154, John Wiley \& Sons, Hoboken, NJ, USA, 2005.

[6] S. Saunders, Antennas and Propagation for Wireless Communication Systems, John Wiley \& Sons, Chichester, UK, 2005.

[7] A. E. Forooshani, S. Bashir, D. G. Michelson, and S. Noghanian, "A survey of wireless communications and propagation modeling in underground mines," IEEE Communications Surveys and Tutorials, vol. 15, no. 4, pp. 1524-1545, 2013.
[8] A. Hrovat, G. Kandus, and T. Javornik, "A survey of radio propagation modeling for tunnels," IEEE Communications Surveys and Tutorials, vol. 16, no. 2, pp. 658-669, 2014.

[9] A. Taflove and S. C. Hagness, Computational Electrodynamics: The Finite-Difference Time-Domain Method, Artech House, Norwood, Mass, USA, 3rd edition, 2005.

[10] M. M. Rana and A. S. Mohan, "Segmented-locally-one-dimensional-FDTD method for em propagation inside large complex tunnel environments," IEEE Transactions on Magnetics, vol. 48, no. 2, pp. 223-226, 2012.

[11] A. G. Emslie, R. L. Lagace, and P. F. Strong, "Theory of the propagation of UHF radio waves in coal mine tunnels," IEEE Transactions on Antennas and Propagation, vol. 23, no. 2, pp. 192-205, 1975.

[12] J. R. Wait, "Propagation in rectangular tunnel with imperfectly conducting walls," Electronics Letters, vol. 16, no. 13, pp. 521-522, 1980.

[13] M. H. Kermani and M. Kamarei, "Ray-tracing method for predicting delay spread in tunnel environments," in Proceedings of the IEEE International Conference on Personal Wireless Communications, pp. 538-542, December 2000.

[14] A. Hrovat, G. Kandus, and T. Javornik, "Four-slope channel model for path loss prediction in tunnels at $400 \mathrm{MHz}$," IET Microwaves, Antennas and Propagation, vol. 4, no. 5, pp. 571$582,2010$.

[15] K. Guan, Z. Zhong, B. Ai, R. He, and C. Briso-Rodríguez, "Fivezone propagation model for large-size vehicles inside tunnels," Progress in Electromagnetics Research, vol. 138, pp. 389-405, 2013.

[16] H. H. Xia, H. L. Bertoni, L. R. Maciel, A. Lindsay-Stewart, and R. Rowe, "Radio propagation characteristics for line-ofsight microcellular and personal communications," IEEE Transactions on Antennas and Propagation, vol. 41, no. 10, pp. 14391447, 1993.

[17] The Ekahau A4 Asset Tag Datasheet, http://www.ekahau .com/userData/ekahau/documents/datasheets/Ekahau_RTLS_ A4_DS.pdf.

[18] Y. P. Zhang, "Novel model for propagation loss prediction in tunnels," IEEE Transactions on Vehicular Technology, vol. 52, no. 5, pp. 1308-1314, 2003.

[19] "Least-Squares (Model Fitting) Algorithms in MATLAB," http://www.mathworks.se/help/optim/ug/least-squares-modelfitting-algorithms.html.

[20] Kristineberg Mine, http://www.mindat.org/loc-13955.html. 


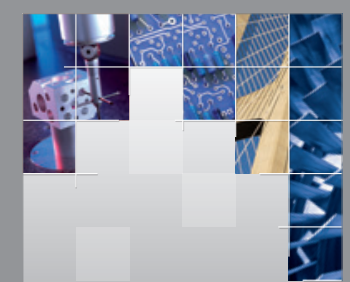

\section{Enfincering}
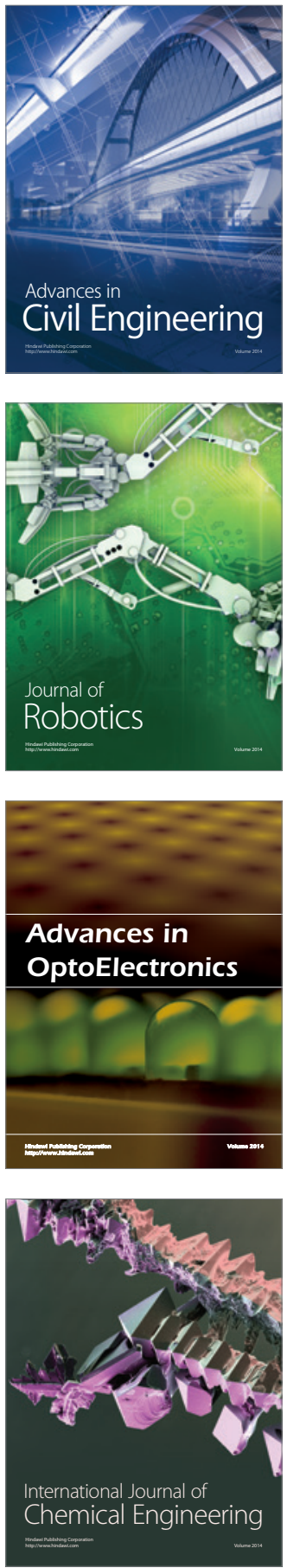

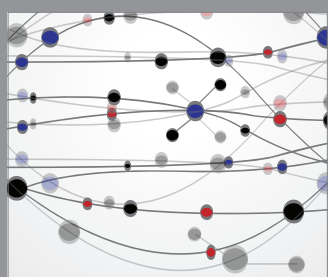

The Scientific World Journal

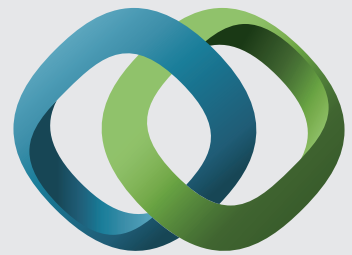

\section{Hindawi}

Submit your manuscripts at

http://www.hindawi.com
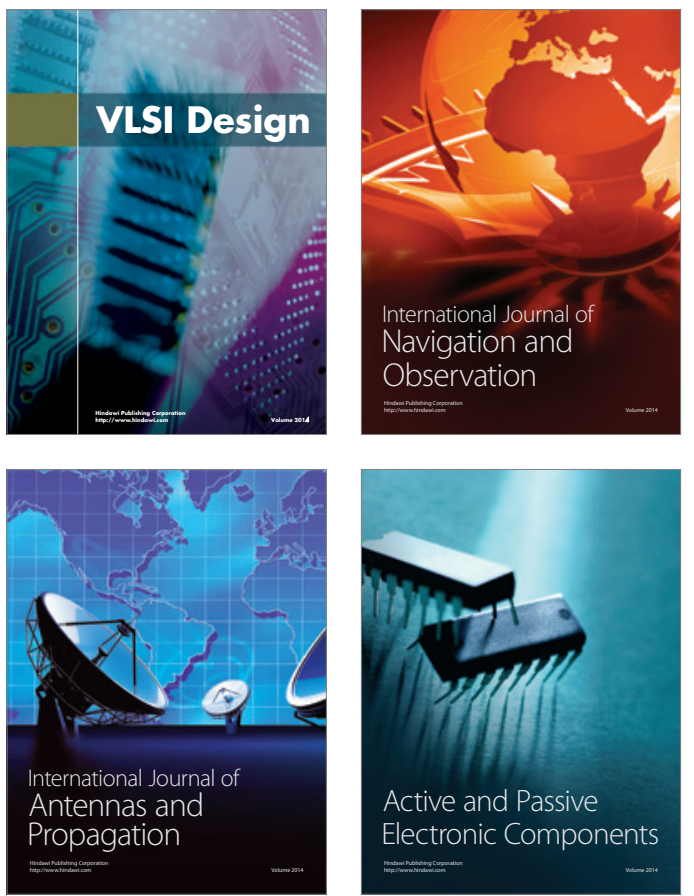
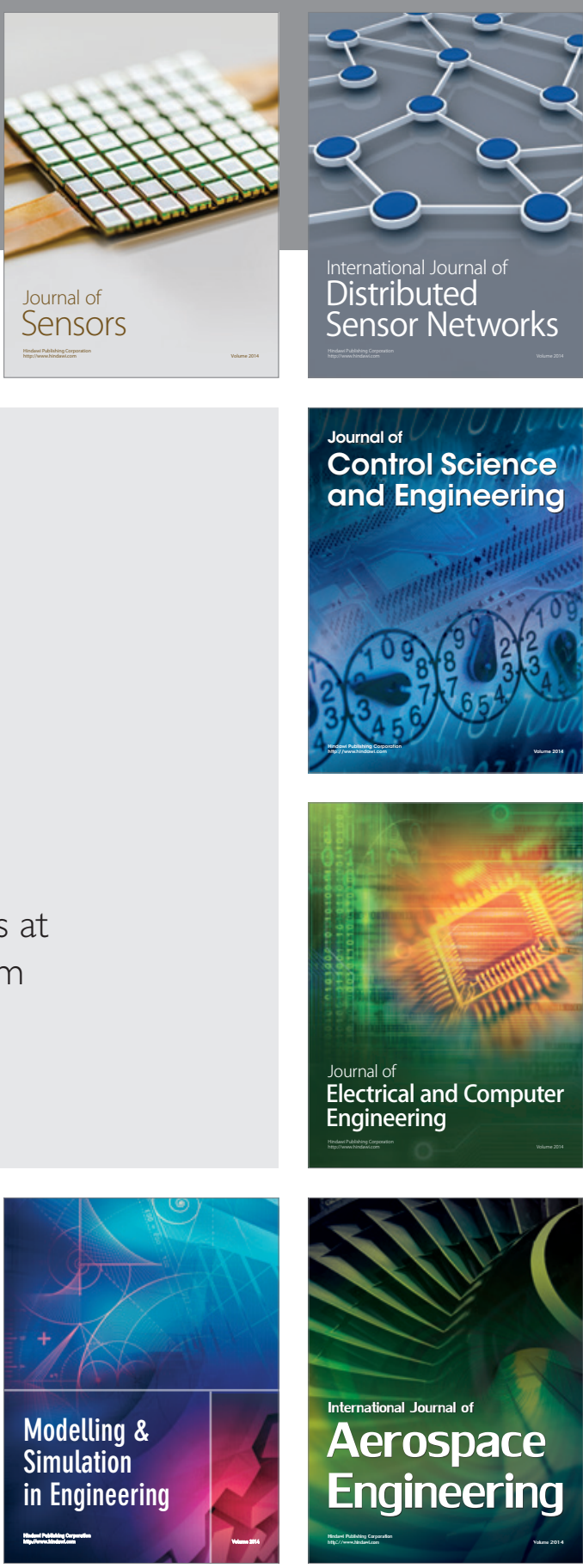

International Journal of

Distributed

Sensor Networks

Journal of

Control Science

and Engineering
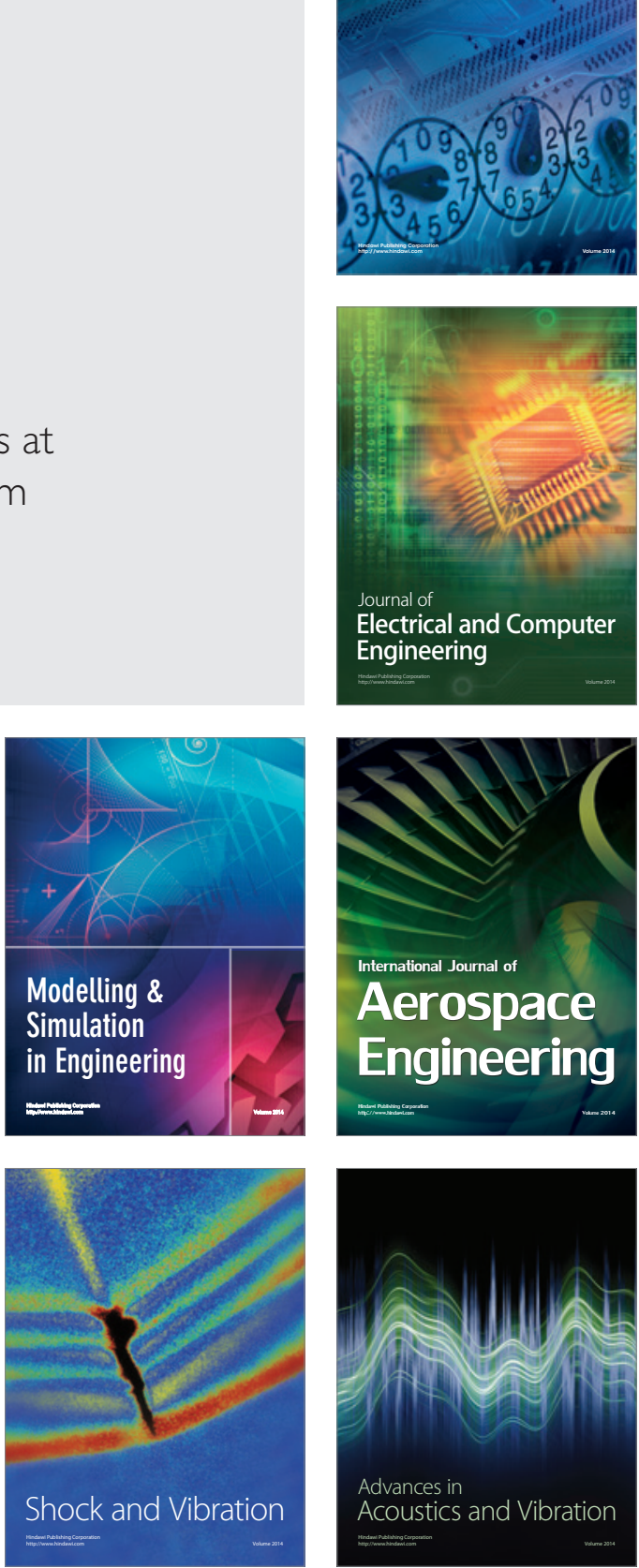